\title{
Imbibition with swelling: Capillary rise in thin deformable porous media
}

\author{
Mathias Kvick and D. Mark Martinez \\ Department of Chemical and Biological Engineering, University of British Columbia, \\ Vancouver, British Columbia, Canada V6T 1Z4 \\ Duncan R. Hewitt \\ Department of Applied Mathematics and Theoretical Physics, University of Cambridge, \\ Cambridge CB3 OWA, United Kingdom
}

Neil J. Balmforth

Department of Mathematics, University of British Columbia, Vancouver, British Columbia, Canada V6T 1Z2

(Received 7 March 2017; published 21 July 2017)

\begin{abstract}
The imbibition of a liquid into a thin deformable porous substrate driven by capillary suction is considered. The substrate is initially dry and has uniform porosity and thickness. Two-phase flow theory is used to describe how the liquid flows through the pore space behind the wetting front when out-of-plane deformation of the solid matrix is considered. Neglecting gravity and evaporation, standard shallow-layer scalings are used to construct a reduced model of the dynamics. The model predicts convergence to a self-similar behavior in all regions except near the wetting front, where a boundary layer arises whose structure narrows with the advance of the front. Over time, the rise height approaches the similarity scaling of $t^{1 / 2}$, as in the classical Washburn or BCLW law. The results are compared with a series of laboratory experiments using cellulose paper sheets, which provide qualitative agreement.
\end{abstract}

DOI: 10.1103/PhysRevFluids.2.074001

\section{INTRODUCTION}

The imbibition of a liquid into a solid matrix has a rich history dating back to Bell and Cameron [1], Lucas [2], and Washburn [3]. The problem involves the interplay between capillary pressure and the viscous drag experienced as the fluid flows through the conduits of the solid medium. Classical scaling analysis leads to the so-called Washburn law (herein referred to as BCLW), which predicts that the penetration length $\ell$ advances diffusively in time,

$$
\frac{\ell^{2}}{t}=\frac{\alpha k_{0} P_{c}}{\mu}
$$

where $\alpha$ is a dimensionless constant, $k_{0}$ is a characteristic permeability, $P_{c}$ is the capillary pressure at the advancing dry line, and $\mu$ is the fluid viscosity. The utility of this relationship has been demonstrated over the years on a wide range of media, including substrates with nanoscopic or complex pore structures. For a porous matrix with a characteristic pore scale of $r_{p}$, one expects that $P_{c} \sim \gamma \cos \theta / r_{p}$, where $\gamma$ is the surface tension of water and $\theta$ the contact angle between the water and solid (see [4] and references therein).

In this paper we study imbibition into a deformable porous substrate. The motivation behind this work stems from an industrial application, namely, the absorbency of paper products used for household, medical, or diagnostic applications [5-10]. Of interest is the interaction between the invading liquid and the (out-of-plane) deformation of the substrate. This problem becomes particularly complex when the substrate is constructed with multiple plies or is initially textured through mechanical embossing or added roughness elements.

A second motivation stems from understanding the wetting behavior of natural cellulose fibers. One interesting feature of this material is that individual fibers swell during imbibition [11]. The 
chemistry of cellulose itself aids in this process: There are numerous (hydrophillic) hydroxl groups in the macromolecule and swelling results as the fluid is able to penetrate into the amorphous regions of the fiber wall [6]. In the classical treatment of liquid penetration into paper, the voids between fibers are viewed as a network of interconnected capillaries and the liquid front advances under the action of capillary forces in both the external pores and fractures in the fiber walls. In one extreme, with very dense paper, Bristow [12] demonstrates that liquid penetration occurs primarily by transport through the fiber walls. Nevertheless, the penetration length has still been found to follow a rough power-law relationship $\ell \sim t^{n}$, with $0.42<n<0.5$ [1,2,13-15]. The suggestion of a slight deviation from classical diffusive behavior with $n=0.5$ becomes further exaggerated when small amounts of superabsorbent materials, such as carboxymethyl cellulose, are mixed into the cellulose sheet, or if the paper is heated, cooled, or exposed to a steam environment during imbibition [15-17]. It has been postulated that this deviation results from swelling of the cellulose matrix and various ad hoc adjustments have been made to the BCLW law to account for the structural changes [18-21].

In this paper we explore the effect of swelling, or out-of-plane deformation, in a thin solid matrix on the dynamics of imbibition into the matrix. A qualitative example of this behavior is shown in Fig. 1(a). Our study combines a theoretical model based on two-phase flow through a deformable porous medium with experiments of imbibition in paper sheets. For the theoretical model (Secs. II and III), we develop previous work on capillary imbibition [22-25] by adding explicitly the out-of-plane dynamics of swelling, within the confines of a shallow-layer description. We further include a rate-dependent rheology to describe the deformation of the porous matrix. While such a rheology has been previously examined in pressure filtration studies of cellulose fiber suspensions [26], its potential effect on the behavior during imbibition has not yet been explored. We investigate the implications of both out-of-plane swelling and rate-dependent solid rheology, but find that neither can account for subdiffusive imbibition.

For our experiments, discussed in Sec. IV, we investigate the imbibition of water into a variety of different types of paper. We monitor the penetration length for a comparison with the BCLW law. We also use optical coherence tomography to measure directly the variation in the thickness of the paper samples during the swelling process. These experiments allow us to characterize in detail the evolution of the swelling front and compare qualitatively with the theoretical results.

\section{MODEL FORMULATION}

We consider flow through a long, thin, two-dimensional, deformable solid porous medium as sketched in Fig. 1(b). The porous layer is initially unsaturated and has uniform thickness $2 H_{0}$ and porosity $\phi_{0}$. At $t=0$, the base of the layer is placed in contact with a reservoir of fluid at $x=0$. The subsequent wicking height, or rise height of the water, is denoted by $x=\ell(t)$. The pore-averaged velocity of the fluid and solid phases are $\boldsymbol{u}_{f}=\left(u_{f}, w_{f}\right)$ and $\boldsymbol{u}_{s}=\left(u_{s}, w_{s}\right)$, respectively. During imbibition, the porosity of the medium is given by $\phi(x, z, t)$ and, assuming that it remains symmetrical about its midline $z=0$, the sides of the porous medium swell out to $z= \pm H(x, t)$. The imbibition is driven by capillary suction and the fluid is assumed to partially wet the medium with a contact angle $0<\theta<\pi / 2$ such that the driving capillary pressure $P_{c}>0$.

Under the assumption that the densities of the solid and fluid phases are constant, the continuity equations for each phase are

$$
\begin{gathered}
\frac{\partial \phi}{\partial t}+\nabla \cdot\left(\phi \boldsymbol{u}_{f}\right)=0, \\
\frac{\partial(1-\phi)}{\partial t}+\nabla \cdot\left[(1-\phi) \boldsymbol{u}_{s}\right]=0 .
\end{gathered}
$$

The relative fluid velocity $\boldsymbol{u}_{f}-\boldsymbol{u}_{s}$ is related to gradients in liquid pressure $p$ through Darcy's law

$$
\phi\left(\boldsymbol{u}_{f}-\boldsymbol{u}_{s}\right)=-\frac{k(\phi)}{\mu} \nabla p,
$$




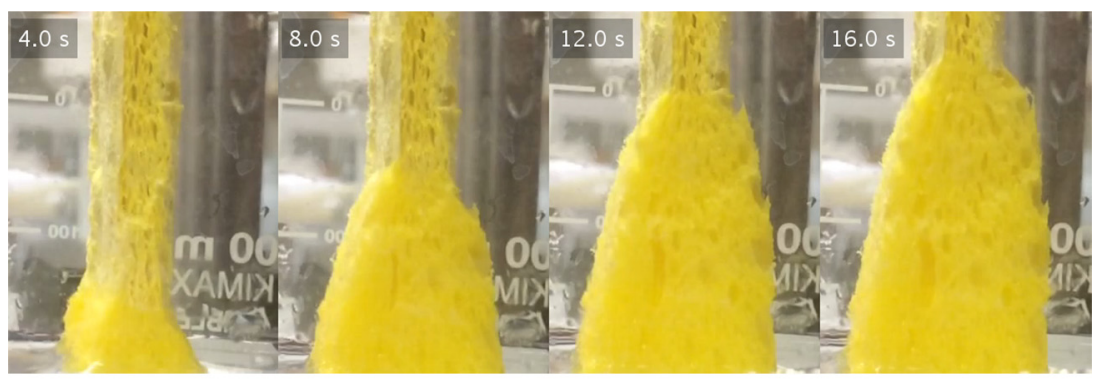

(a)

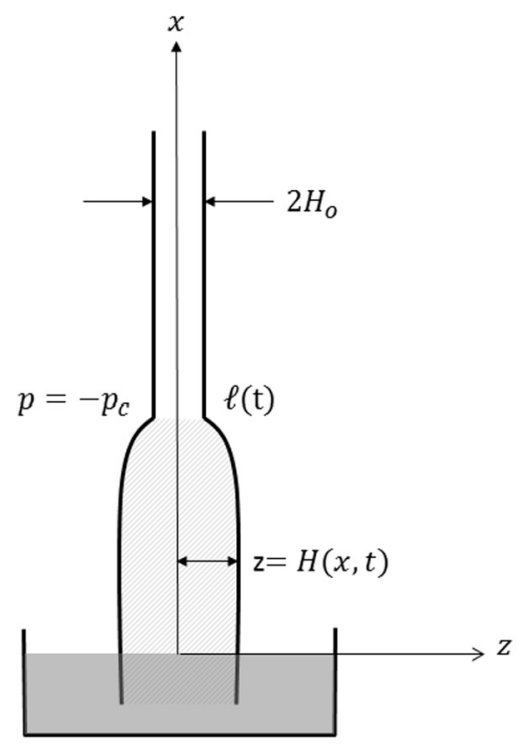

(b)

FIG. 1. Illustration of the imbibition problem. (a) Example snapshots over time, as marked, of imbibition into a swelling cellulose sponge. For reference, the initial half-width of the sponge is $5 \mathrm{~mm}$. (b) Sketch of the geometry viewed from the side and the definition of the variables. The shaded area represents the wet portion of the porous media.

where $\mu$ is the fluid viscosity and $k(\phi)$ is the permeability, which is taken to be a function of the local porosity. With the neglect of gravity and inertia, force balance on the bulk medium indicates that

$$
\nabla \cdot \sigma-\nabla p=0
$$

where $\boldsymbol{\sigma}$ is the excess network stress tensor for the fluid-solid mixture. The neglect of gravity in the model remains reasonable for as long as the buoyancy force associated with the imbibed fluid remains small relative to the driving capillary pressure.

To close the equations above we must specify $\boldsymbol{\sigma}$. This is often accomplished by adopting a constitutive law in which $\sigma$ is dominated by its isotropic component, which is then given as a prescribed function of the local solidity, i.e., $\boldsymbol{\sigma}=\sigma \mathbf{I} \equiv \sigma_{e}(\phi) \mathbf{I}$, for some suitable constitutive function $\sigma_{e}(\phi)$ (see, e.g., [23,24]). Indeed, even a more general stress tensor $\sigma$ can reduce to this isotropic form in certain asymptotic limits (e.g., a long, thin lubrication limit, as in [27]).

However, there is some speculation that describing cellulose rheology purely in terms of the local solid fraction may not be adequate, owing to the swelling of cellulose fibers on the microscale, 
which is plausibly a rate-dependent process. We therefore adopt a rheology similar to that originally suggested by Buscall and White [28], for which $\boldsymbol{\sigma}=\sigma \mathbf{I}$ and

$$
\begin{aligned}
\sigma-\sigma_{e}(\phi) & =(1-\phi) \Lambda(\phi) \nabla \cdot \boldsymbol{u}_{s} \\
& =-(1-\phi) \Lambda(\phi)\left(\frac{\partial \phi}{\partial t}+\boldsymbol{u}_{s} \cdot \nabla \phi\right),
\end{aligned}
$$

where the bulk viscosity function $\Lambda(\phi)$ may depend on the permeability and elasticity of the fiber wall and the fluid viscosity. This expression reduces to $\sigma=\sigma_{e}(\phi)$ in the limit $\Lambda \ll 1$ and provides a simple model of rate-dependent relaxation towards that equilibrium stress, not unlike a Kelvin-Voigt model for a viscoelastic solid. It has been recently adopted by Hewitt et al. [26] to describe the compression of cellulose fiber suspensions during one-dimensional pressure filtration and was shown to give a dramatic qualitative improvement to model predictions, relative to the simple instantaneous model $\sigma=\sigma_{e}(\phi)$, in that case. Note that we have not included any mass transfer terms in the continuity equations (2) and (3) as a result of swelling, in line with the two-phase idealization of the problem, which does not distinguish between the water inside and outside the fibers. This formulation requires revision if the fluid interior of the fibers is, on the time scale of imbibition, effectively isolated from that outside those solid structures. A biporosity model would then be needed, along with mass transfer terms in the two fluid continuity equations.

The boundary conditions are as follows. The sheet remains symmetric about the centerline, so that

$$
w_{s}(x, 0, t)=w_{f}(x, 0, t)=0 .
$$

On the side surface, $z=H(x, t)$, kinematic conditions for fluid and solid phases imply

$$
\begin{aligned}
& w_{f}(x, H, t)=\frac{\partial H}{\partial t}+u_{f}(x, H, t) \frac{\partial H}{\partial x} \\
& w_{s}(x, H, t)=\frac{\partial H}{\partial t}+u_{s}(x, H, t) \frac{\partial H}{\partial x}
\end{aligned}
$$

while the total stress vanishes there,

$$
\sigma(x, H, t)-p(x, H, t)=0 .
$$

These conditions imply that neither infiltration of air through this surface nor leakage of fluid across the surface can occur; we note that leakage was not observed in any of our experiments.

At the surface of the fluid reservoir $(x=0)$, the fluid pressure is atmospheric (and taken to be zero):

$$
p(0, z, t)=0 .
$$

At the dry line $x=\ell(t)$, the fluid pressure must balance the capillary pressure $-P_{c}$ and the thickness of the sheet must match to its original value

$$
\begin{aligned}
& p(\ell, z, t)=-P_{c} \\
& \phi(\ell, z, t)=\phi_{0} .
\end{aligned}
$$

\section{A. Shallow-layer scalings}

We now suppose that the thickness $H_{0}$ of the porous layer is much less than the length scale $L_{0}$ characterizing variation along its length, so $\delta \equiv H_{0} / L_{0} \ll 1$. We then introduce the rescalings 
(cf. [27])

$$
\begin{aligned}
\left(x^{*}, \ell^{*}\right) & =\frac{1}{L_{0}}(x, \ell), \quad\left(p^{*}, \sigma^{*}\right)=\frac{1}{P_{c}}(p, \sigma), \\
\left(z^{*}, H^{*}, t^{*}, h_{s}^{*}\right) & =\frac{1}{H_{0}}\left(z, H, U_{c} t, h_{s}\right), \quad u_{f}^{*}=\frac{\delta u_{f}}{U_{c}}, \\
\left(w_{f}^{*}, u_{s}^{*}, w_{s}^{*}\right) & =\frac{1}{U_{c}}\left(w_{f}, u_{s}, w_{s}\right),
\end{aligned}
$$

where

$$
U_{c}=\frac{\delta^{2} k_{0} P_{c}}{\mu H_{0}}
$$

is a characteristic velocity based on the main balance in Darcy's law and $k_{0}$ is a characteristic measure of the permeability. Here the different choice of scaling for $u_{f}$ and $w_{f}$ follows conventional assumptions for a shallow film and is designed to accommodate all terms in the fluid continuity equation. The same arguments, however, are difficult to carry over to the components of the solid velocity, in view of the detailed solid mechanics that can prevent significant in-plane displacements. Indeed, Kulachenko [29] argues that paper swells predominately in the out-of-plane direction during wetting and there is negligible expansion in the in-plane direction, even when the sample is unconstrained. Thus, we assume that the magnitude of the in-plane deformation is of the order of the out-of-plane displacement or smaller.

With the scalings in (12), the dimensionless continuity equations (2) and (3), Darcy's law (4), and force balance (5) are, to leading order in $\delta$,

$$
\begin{gathered}
\frac{\partial \phi}{\partial t^{*}}=\frac{\partial}{\partial z^{*}}\left[(1-\phi) w_{s}^{*}\right]=-\frac{\partial}{\partial x^{*}}\left(\phi u_{f}^{*}\right)-\frac{\partial}{\partial z^{*}}\left(\phi w_{f}^{*}\right), \\
-\phi u_{f}^{*}=k^{*}(\phi) \frac{\partial p^{*}}{\partial x^{*}}, \quad \frac{\partial p^{*}}{\partial z^{*}}=0
\end{gathered}
$$

and

$$
\frac{\partial}{\partial x^{*}}\left(\sigma^{*}-p^{*}\right)=\frac{\partial \sigma^{*}}{\partial z^{*}}=0
$$

where $k^{*}=k(\phi) / k_{0}$. The closure relation (6), with Eq. (3), becomes

$$
\sigma^{*}=p_{e}(\phi)+\epsilon \Lambda^{*}(\phi)(1-\phi) \frac{\partial w_{s}^{*}}{\partial z^{*}}
$$

with

$$
\epsilon=\frac{k_{0} \Lambda_{0}}{\mu L_{0}^{2}}, \quad p_{e}(\phi)=\frac{\sigma_{e}(\phi)}{P_{c}}, \quad \Lambda^{*}=\frac{\Lambda(\phi)}{\Lambda_{0}},
$$

where $\Lambda_{0}$ is the characteristic scale of the bulk viscosity function $\Lambda(\phi)$. The parameter $\epsilon$ measures the relative importance of the rate-dependent stresses and provides a ratio of the characteristic time scale for relaxation of the matrix to the time scale for pore-pressure diffusion. In the shallow limit, the dimensionless boundary conditions remain unchanged from their dimensional counterparts in (7)-(11), except for the kinematic condition on the solid (8b), which reduces to

$$
w_{s}^{*}\left(x^{*}, H^{*}, t^{*}\right)=\frac{\partial H^{*}}{\partial t^{*}},
$$

and the dry-line pressure condition (11a), which becomes

$$
p^{*}\left(\ell^{*}, z^{*}, t^{*}\right)=\sigma^{*}\left(\ell^{*}, z^{*}, t^{*}\right)=-1,
$$

in light of (16). 
Note that the in-plane length scale $L_{0}$ is not selected in the relations above. In fact, without the rate-dependent rheological term parametrized by $\epsilon$, this length scale remains free, highlighting how the leading-order system of equations possesses a scaling symmetry and therefore a similarity solution (as detailed further below). The rate-dependent stress term breaks this symmetry and thereby selects a characteristic in-plane length scale; equivalently, we may choose $L_{0}$ to set the parameter $\epsilon$ to unity. However, since this term constitutes a new addition to the model, it is expeditious to retain $\epsilon$ as a parameter, which can be set to zero to recover a formulation similar to previous models [22-25]. We therefore leave $L_{0}$ free, demanding only that the shallow-layer scaling $\delta=H_{0} / L_{0} \ll 1$ is satisfied.

\section{B. Dimensionless model}

We now drop the asterisk notation that indicates a dimensionless quantity and simplify the leading-order system of equations to construct a reduced model. We first note that Eqs. (16) and (17) are satisfied and consistent with the initial condition $\phi(x, z, 0)=\phi_{0}$ and boundary conditions if

$$
p=p(x, t)=\sigma=\sigma(x, t), \quad \phi=\phi(x, t)
$$

and

$$
w_{s}=\frac{z}{H} \frac{\partial H}{\partial t} .
$$

The continuity and kinematic surface conditions can then be combined into the thickness-averaged mass conservation equations

$$
\begin{gathered}
\frac{\partial}{\partial t}(\phi H)+\frac{\partial}{\partial x}\left(\phi u_{f} H\right)=0, \\
\frac{\partial}{\partial t}[(1-\phi) H]=0 .
\end{gathered}
$$

Upon integration, and in view of the initial conditions, Eq. (24) furnishes

$$
(1-\phi) H=1-\phi_{0},
$$

which relates the porosity $\phi$ and thickness $H$ in terms of the initial porosity $\phi_{0}$. Thence, from (15), (23), and (17),

$$
\begin{aligned}
\frac{\partial H}{\partial t} & =\frac{\partial}{\partial x}\left[k(\phi) H \frac{\partial \sigma}{\partial x}\right] \\
\sigma & =p_{e}(\phi)+\epsilon \Lambda(\phi) \frac{(1-\phi)}{H} \frac{\partial H}{\partial t}
\end{aligned}
$$

subject to

$$
\begin{gathered}
H(x, 0)=H_{\ell}(t)=1 \quad\left[\text { or } \phi(x, 0)=\phi_{\ell}(t)=\phi_{0}\right], \\
\sigma(0, t)=0, \quad \sigma(\ell, t)=-1,
\end{gathered}
$$

where $H_{\ell}(t)=H(\ell, t)$ and $\phi_{\ell}=\phi(\ell, t) \equiv 1-\left(1-\phi_{0}\right) / H_{\ell}$. We further note the kinematic dry-line condition $d \ell / d t=\phi u_{f}(\ell, t)$ or

$$
\frac{d \ell}{d t}=-\left.k(\phi) \frac{\partial \sigma}{\partial x}\right|_{x=\ell},
$$

with the initial condition $\ell(0)=0$. 


\section{Solution method}

Given the parameters $\phi_{0}$ and $\epsilon$, together with the constitutive functions $p_{e}(\phi), \Lambda(\phi)$, and $k(\phi)$ (discussed below), the evolution of $H(x, t)$ is given by (26) on a domain $[0<x<\ell]$, with $\ell(t)$ given by (29). In order to solve this problem numerically, we first map the problem onto a fixed domain by means of the transformation $y=x / \ell(t)$, exploiting the relation (29).

If $\epsilon>0$, we solve (26) as an elliptic problem for $\sigma(y, t)$ at each time step, together with a hyperbolic evolution equation for $H(y, t)$ [or $\phi(y, t)$ ] and a simple ordinary differential equation (ODE) for $\ell(t)$. The boundary and initial conditions are given in (27) and (28). We use a standard second-order finite-difference discretization in space and a fourth-order Runge-Kutta scheme to evolve in time. In order to regularize the numerical scheme at very early times, we replace the initial condition $\ell(0)=0$ by $\ell(0)=\ell_{0}$, for some small positive $\ell_{0}$ (in practice, we typically take $\ell_{0}=10^{-3}$, but find its value has no appreciable effect on solutions at subsequent times).

In the distinguished limit $\epsilon=0$, the formulation instead takes the form of a nonlinear diffusion equation for $H(y, t)$, which has a similarity solution as discussed in Sec. III A below. We determine this solution using MATLAB's built-in bvp4c routine.

\section{Constitutive functions}

To provide illustrative solutions of the model, we will use simple constitutive laws that describe the basic qualitative behavior that we might expect for any deformable medium. For the rate-independent part of the effective stress, we adopt the simple linear form

$$
p_{e}(\phi)=\frac{\phi-\phi_{r}}{\phi_{r}-\phi_{c}}
$$

(cf. [22] or classical linear poroelasticity theory), where $\phi_{r}$ and $\phi_{c}$ (with $\phi_{c}<\phi_{r}$ ) are the porosities of the fully relaxed state and for which the rate-independent effective stress equals the capillary pressure, respectively. Note that both $\phi_{r}$ and $\phi_{c}$ are properties of the wet medium and both are larger than the initial dry porosity $\phi_{0}$.

For simplicity, we set the bulk viscosity function $\Lambda(\phi)$ to be constant $\Lambda=1$, while for the permeability we use a standard Kozeny-Carman relationship

$$
k(\phi)=\left(\frac{\phi}{\phi_{r}}\right)^{3}\left(\frac{1-\phi_{r}}{1-\phi}\right)^{2}
$$

(see [30]). Here we have selected the characteristic value $k_{0}$ such that $k=1$ when $\phi=\phi_{r}$.

\section{MODEL RESULTS}

\section{A. The limit $\epsilon \rightarrow 0$ : Similarity solution}

As was noted at the end of Sec. II B, in the limit $\epsilon \rightarrow 0$ the in-plane length scale $L_{0}$ is left free, which implies a scaling symmetry and a similarity solution. The conditions at the wetting front $x=\ell(t)$ and water reservoir $x=0$ must also be modified in this limit: If $\epsilon=0$, (28) reduces to

$$
p_{e}\left(\phi_{b}\right)=0, \quad p_{e}\left(\phi_{\ell}\right)=-1,
$$

where $\phi_{b}=\phi(0, t)=1-\left(1-\phi_{0}\right) / H_{b}$. The first of these conditions indicates that the porosity and width at the water reservoir jump immediately to their steady-state values $\phi_{b}=\phi_{r}$ and $H_{b}=$ $\left(1-\phi_{0}\right) /\left(1-\phi_{r}\right)$ in this limit. The second condition in (32) demands that $p_{e}\left(\phi_{\ell}\right)=-1$ such that $\phi_{\ell}=\phi_{c}$ and $H_{\ell} \neq 1$. As a result, there is also an instantaneous jump in the porosity and width at the wetting front $x=\ell$ and the second boundary condition in (27) must be abandoned. 

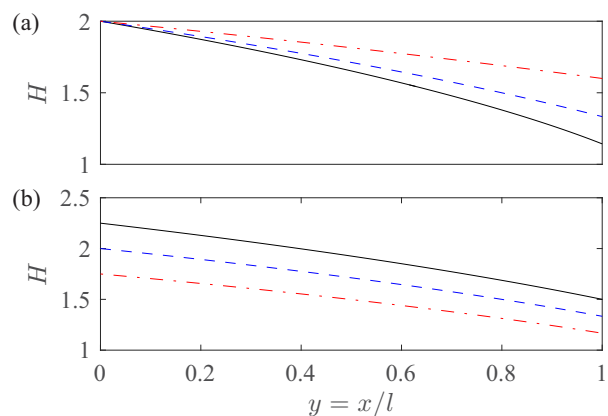

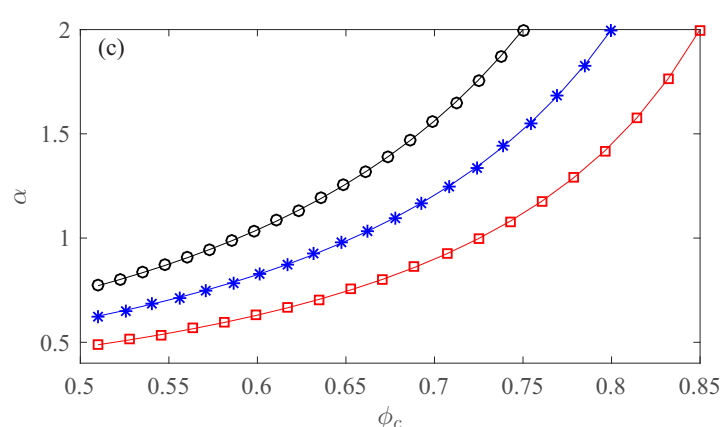

FIG. 2. Sample similarity solutions of the model with $\epsilon=0$. (a) Width $H(y)$ for $\phi_{r}=0.8, \phi_{0}=0.6$, and $\phi_{c}=0.65$ (black solid line), $\phi_{c}=0.7$ (blue dashed line), and $\phi_{c}=0.75$ (red dot-dashed line). (b) Width $H(y)$ for $\phi_{r}=0.8, \phi_{c}=0.7$, and $\phi_{0}=0.55$ (black solid line), $\phi_{0}=0.6$ (blue dashed line), and $\phi_{0}=0.65$ (red dot-dashed line). (c) Parameter $\alpha$ that controls the rate of spreading [see (33)], for $\phi_{r}=0.75$ (black circles), $\phi_{r}=0.8$ (blue stars), and $\phi_{r}=0.85$ (red squares). Note that $\alpha$ is independent of the initial condition $\phi_{0}$.

To find the similarity solution, we set

$$
y=\frac{x}{\ell}, \quad \ell=\sqrt{\alpha t}, \quad \phi=\phi(\eta), \quad H=H(\eta),
$$

where $\alpha$ is a constant that must be determined as part of the solution. Equations (26) reduce to

$$
-\frac{\alpha y}{2\left(1-\phi_{0}\right)} \frac{d H}{d y}=\frac{d}{d y}\left[\frac{k(\phi) p_{e}^{\prime}(\phi)}{H} \frac{d H}{d y}\right],
$$

subject to

$$
\begin{aligned}
\frac{\alpha}{2\left(1-\phi_{0}\right)} & =-\left.\frac{k(\phi) p_{e}^{\prime}(\phi)}{H^{2}} \frac{d H}{d y}\right|_{y=1}, \\
H(0) & =\frac{1-\phi_{0}}{1-\phi_{r}}, \quad H(1)=\frac{1-\phi_{0}}{1-\phi_{c}},
\end{aligned}
$$

where $p_{e}^{\prime}(\phi) \equiv d p_{e} / d \phi$. Solutions of (34) for a variety of parameters are shown in Fig. 2. Since the porosity instantly jumps at the upper and lower edges of the wet region, the initial porosity $\phi_{0}$ plays no role in the solutions other than to set the available solid mass and therefore the scale of the swelling [Fig. 2(b)]. In particular, the rate of advance of $\ell(t)$, as described by the parameter $\alpha$, is independent of $\phi_{0}$ [Fig. 2(c)]. This parameter also decreases as the relaxed porosity $\phi_{r}$ is increased, because the medium can expand further, and increases as $\phi_{c}$ is increased, which corresponds to an increase in the relative stiffness of the medium. Note that in the limit $\phi_{c} \rightarrow \phi_{r}$, the porosity variation is weak $p_{e}^{\prime}=\left(\phi_{r}-\phi_{c}\right)^{-1} \gg 1$ and $k(\phi) \rightarrow 1$. Hence Eqs. (34) and (35) imply that $\phi \sim \phi_{r}-\left(\phi_{r}-\phi_{c}\right) y$, as $\phi_{c} \rightarrow \phi_{r}$, and $\alpha=2$ [see Fig. 2(c)].

\section{B. Numerical solutions for $\epsilon>0$}

Figure 3 shows some sample solutions for computations with $\epsilon>0$. Even though a self-similar evolution is now no longer expected, the computations show that the width $H(y, t)$ still evolves from its initial value of $H=1$ and approaches the $\epsilon=0$ similarity solution everywhere except near the wetting front $y=1$ [Fig. 3(a)]. Here the failure of the similarity solution to satisfy the boundary condition leads to an increasingly narrow boundary layer over which the width profile deviates from the similarity form and matches to $H=1$.

As a consequence of this evolution, the wetting front $\ell(t)$ initially rises slower than the prediction $\ell=\sqrt{\alpha t}$ [Fig. 3(b)]. However, as the profiles evolve towards the similarity profile, $\ell$ converges to 

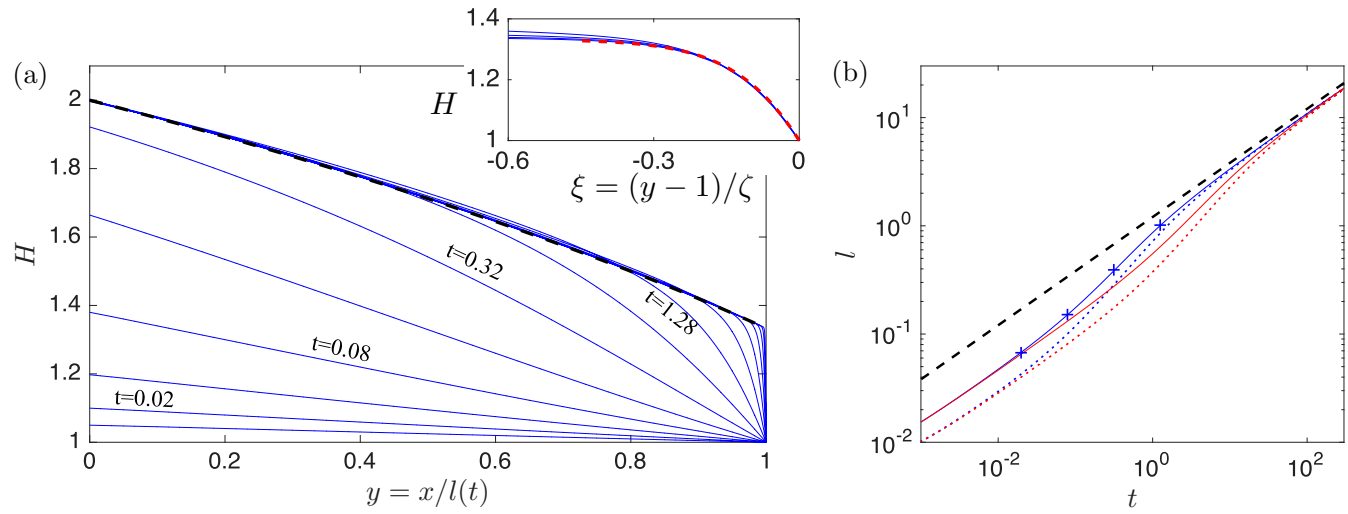

FIG. 3. Sample solutions for $\phi_{r}=0.8, \phi_{c}=0.7$, and $\epsilon>0$. (a) Snapshots of $H(y, t)$ for $\epsilon=1$ and $\phi_{0}=0.6$, at times $t=0.02 \times 2^{n}$ for integer $n \geqslant 0$, together with the corresponding similarity solution for $\epsilon=0$ (black dashed line). The inset shows the nose region rescaled as in (38) for four snapshots at late times (between $t=10$ and $t=80$ ), together with the asymptotic solution from (41) (red dashed). (b) Rise height $\ell(t)$ for $\epsilon=1$ (blue lines) and $\epsilon=10$ (red lines) and $\phi_{0}=0.6$ (solid lines) and $\phi_{0}=0.5$ (dotted lines). The thick dashed line shows the corresponding similarity solution (which does not depend on $\phi_{0}$ ). The marked times in (a) correspond to the crosses in (b).

$\sqrt{\alpha t}$. Evidently, over sufficiently long times, the boundary-layer structure that forms near the wetting front has a negligible effect on the rise height. In fact, we demonstrate in the following section that this behavior is a generic feature of the dynamics captured by the model, irrespective of the choice of constitutive functions.

Figure 3(b) also shows that, as well as evolving towards the similarity solutions, the wetting front also follows a scaling of $t^{1 / 2}$ at early times, but with a smaller prefactor. In fact, an analysis of (26) with $t \ll \epsilon$ suggests a scaling of

$$
H=1+t \epsilon^{-1} \hat{H}(x / \sqrt{t}),
$$

in which limit the equations again reduce to an ODE for $\hat{H}$, to leading order in $t / \epsilon$. Thus the model predicts an early-time similarity solution, followed by evolution from one scaling of $t^{1 / 2}$ towards another, with the transition between the two regimes occurring after a time $t=O(\epsilon)$.

\section{Approach to the self-similar form}

To rationalize the approach to self-similar form observed in the previous section, we consider the limit $t \gg O(\epsilon)$ in the model (26). We work in a fixed domain by setting $y=x / \ell(t)$, such that $H=H(y, t)$, and define $\zeta(t) \equiv \epsilon \dot{\ell} / \ell$. In the limit $\zeta \ll 1$, the solutions satisfy

$$
\begin{gathered}
\sigma \sim p_{e}+O(\zeta), \\
-y \ell \dot{\ell} \frac{\partial H}{\partial y} \sim \frac{\partial}{\partial y}\left(k p_{e}^{\prime} H \frac{\partial \phi}{\partial y}\right)+O(\zeta),
\end{gathered}
$$

where $\dot{\ell} \equiv d \ell / d t$, provided gradients in $H$ (or $\phi$ ) are relatively gentle [i.e., of $O(1)$ or less]. Based on the numerical results in Fig. 3(a), we expect this to be a valid assumption in the outer region away from the wetting front at $y=1$. Within the boundary layer near $y=1$, however, we rescale to resolve the sharp variation of $\phi$ from $\phi_{c}$ to $\phi_{0}$, by setting

$$
\xi=\frac{y-1}{\zeta}
$$


in which case

$$
\begin{array}{r}
-\frac{\partial H}{\partial \xi} \sim \frac{\left(\sigma-p_{e}\right) H}{(1-\phi) \Lambda}, \\
\frac{\partial}{\partial \xi}\left(k H \frac{\partial \sigma}{\partial \xi}\right) \sim 0
\end{array}
$$

to leading order in $\zeta$, from (26), and assuming $\epsilon \dot{\zeta} / \zeta$ is small. Both this and the original assumption $\zeta \ll 1$ can be verified $a$ posteriori provided $t \gg 1$. In view of the boundary condition $\sigma(\ell, t)=-1$ and the kinematic condition (29), (39bb) integrates to

$$
\sigma \sim-1+\zeta \sigma_{1}
$$

Thus, (39b a) can be integrated to give an implicit equation for the porosity through the boundary layer

$$
\xi=\int_{\phi_{0}}^{\phi} \frac{\Lambda(\varphi) d \varphi}{1+p_{e}(\varphi)}
$$

or, given (25), for the width $H(\xi)$. The next-order correction to the stress can also be calculated from the $O(\zeta)$ balance in $(39 \mathrm{~b} \mathrm{~b})$,

$$
k H \frac{\partial \sigma_{1}}{\partial \xi}+H \ell \dot{\ell} \sim 0
$$

or

$$
\sigma_{1}=-\ell \dot{\ell} \int_{0}^{\xi} \frac{d \xi}{k(\phi)}=-\ell \dot{\ell} \int_{\phi_{0}}^{\phi} \frac{\Lambda(\varphi) d \varphi}{\left[1+p_{e}(\varphi)\right] k(\varphi)}
$$

using (41).

Irrespective of the detailed boundary-layer solution, we now observe that the outer problem in (37b) must obey the matching conditions $\sigma \sim p_{e}(\phi) \rightarrow-1$ (or $\phi \rightarrow \phi_{c}$ ) and $k(\phi) p_{e}^{\prime}(\phi) \phi_{x} \rightarrow \dot{\ell}$ as $x \rightarrow \ell$, which are identical to the boundary conditions imposed on the $\epsilon=0$ similarity solution if $2 \ell \dot{\ell} \equiv \alpha$. Thus, given that (37b) reduces to (34) to leading order in $\zeta$, the solution for the outer region is just the similarity solution with $\epsilon=0$ and $\ell=\sqrt{\alpha t}$. The small parameter $\zeta$ is therefore $\zeta \equiv \epsilon \dot{\ell} / \ell=\epsilon t^{-1} / 2$ and the boundary-layer width shrinks like $t^{-1}$ in similarity space or like $t^{-1 / 2}$ in terms of the true spatial coordinate $x$ [and $\epsilon \dot{\zeta} / \zeta=O\left(t^{-1}\right)$ ]. The height across the nose region predicted by (41) is shown in the inset of Fig. 3(a), together with a selection of snapshots that collapse onto this profile.

We thus conclude that, even with a rate-dependent rheology, the model predicts that imbibition of a thin deformable sheet evolves towards the classical self-similar form predicted by the BCLW law (1). Note that the model must eventually break down, as the shallow layer approximation is violated once the the width of the thinning boundary layer becomes comparable to the thickness of the sheet.

\section{LABORATORY EXPERIMENTS}

In this section we present the results of our laboratory experiments and compare with some of the predictions of the model. For this task we developed a number of different experimental protocols, which are explained alongside the corresponding result.

\section{A. Paper structure}

We used water and a variety of different paper sheets as our experimental materials and we begin by briefly discussing the three-dimensional structure of the sheets. We constructed a 

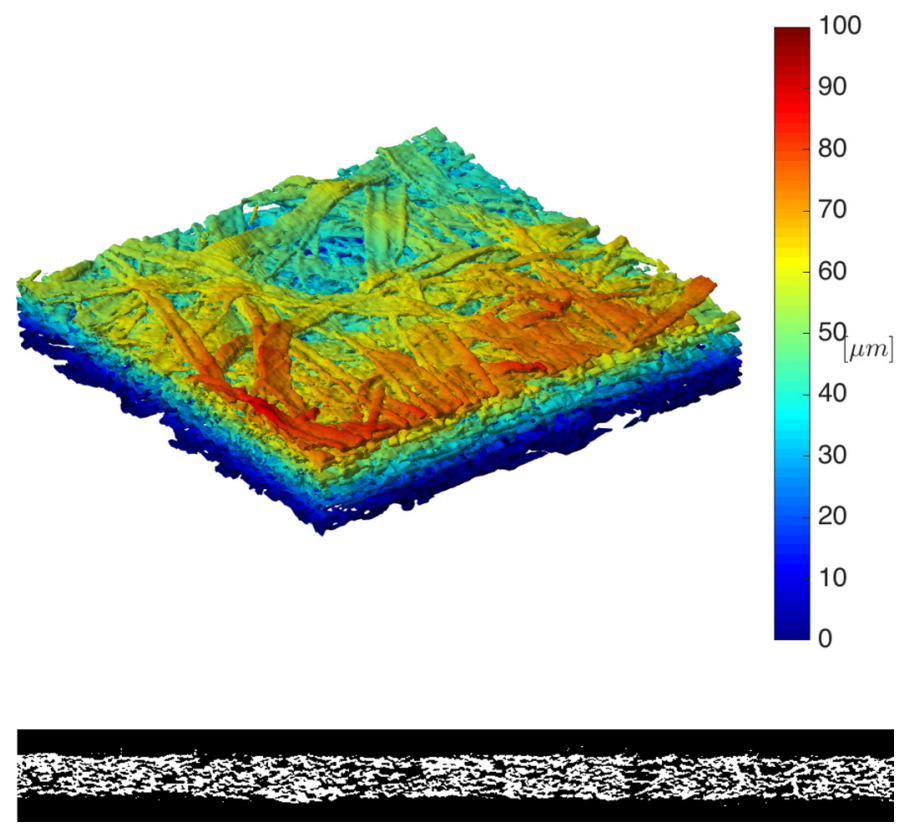

FIG. 4. Computed $x$-ray $\mu$ tomography of an NBSK sheet. In the top image, we show a reconstructed image to highlight the surface and thickness variations $H(x, y)$ in the sheet. The color bar represents the local thickness. In the lower panel a binarized cross section of this sheet is shown. Specifications of the sheet are recorded as series 1 in Table I.

three-dimensional image of a paper sample $\left(1.0 \times 1.0 \mathrm{~mm}^{2}\right.$ in cross section) by performing $\mathrm{x}$-ray tomographic microscopy using a Zeiss MicroXCT-400. Using the methodology outlined by Sharma et al. [31], $2500 \mathrm{x}$-ray radiographs were acquired, at different planes spanning $360^{\circ}$, at a $30-\mathrm{s}$ exposure time, using a 40-kV x-ray source. A long exposure time was required to offset the poor contrast at the fiber-air interfaces. From these radiographs, a three-dimensional volume consisting of $2000 \times 2000 \times 300$ voxels was reconstructed with a resolution of $0.58 \mu \mathrm{m}$ per voxel. An example image is shown in Fig. 4. From this image we can see that fibers form a network of interconnected capillaries that provide flow channels. It is also evident that the fiber axis is generally aligned with the plane.

From these images we can extract a representative measure of the typical pore size $r_{p}$ (i.e., by measuring the size of the dark areas in the cross section in Fig. 4), from which we could in principle estimate the capillary pressure $P_{c} \sim \gamma \cos \theta / r_{p}$ and permeability $k_{0} \sim r_{p}^{2}$, given the surface tension of water $\gamma \approx 0.07 \mathrm{~N} / \mathrm{m}$ and $38^{\circ}<\theta<78^{\circ}$ [14]. However, this estimate gives $r_{p} \sim 10^{-5} \mathrm{~m}, P_{c}=O\left(10^{4}\right) \mathrm{Pa}$, and $k_{0}=O\left(10^{-10}\right) \mathrm{m}^{2}$, which is four orders of magnitude higher than the actual measured permeability of pulp (see Table I). We therefore infer that the pore scale suggested in Fig. 4 cannot be the relevant pore length scale of the cellulose matrix. Unfortunately, since the spatial resolution of the radiograph images is not sufficient to resolve the detailed structure of the individual fibers, we can only speculate as to the reason for this large discrepancy; it may be because the complex microstructure of paper leads to a poorly connected network of voids, resulting in a multitude of bottlenecks for flow. Given this evident complexity of the pore scale and in order to generate a rough prediction for the capillary pressure, we instead use the measured permeability to give a crude estimate of the relevant effective pore scale: $r_{p} \sim \sqrt{k_{0}} \sim 10^{-7} \mathrm{~m}$. This leads us to the estimate $P_{c} \sim \gamma / \sqrt{k_{0}}=O\left(10^{7}\right) \mathrm{Pa}$, which is much closer to the capillary pressure required to explain the relatively large penetration lengths found in our imbibition tests. 
TABLE I. Experimental parameters. The values for the initial thickness are reported to one significant figure due to the surface roughness of the sheet. The permeability values are those reported by Hewitt et al. [26]. The variable $m_{r}$ is a measurement of the water content per unit mass of fiber in the fully swollen state. This term is traditionally called the moisture ratio and is proportional to $\phi_{r}$. The uncertainty in $m_{r}$ is estimated to be $9 \%$.

\begin{tabular}{lccccc}
\hline \hline Series & Fiber source & $2 H_{0}(\mathrm{~mm})$ & $k_{0}\left(10^{-14}\right)\left(\mathrm{m}^{2}\right)$ & $\phi_{0}$ & $m_{r}(\mathrm{~g} / \mathrm{g})$ \\
\hline 1 & NBSK & 0.1 & 7 & 0.6 & 0.55 \\
2 & eucalyptus & 0.1 & 1 & 0.6 & 0.53 \\
3 & eucalyptus & 0.2 & 1 & 0.6 & 0.53 \\
4 & aspen & 0.1 & 3 & 0.6 & 0.50 \\
5 & aspen & 0.2 & 3 & 0.6 & 0.50 \\
\hline \hline
\end{tabular}

We used three different types of cellulose fibers to construct different paper sheets: aspen, eucalyptus, and northern bleached softwood kraft (NBSK) pulp. Aspen and eucalyptus are hardwoods having an average fiber length of approximately $0.8 \mathrm{~mm}$; NBSK is a mixture of spruce, fir, and pine (unknown mixture ratio) with a fiber length of approximately $2 \mathrm{~mm}$. In each case, we made the paper sheets with a British Handsheet maker using TAPPI standard methodologies. A summary of the materials used, as well as a selection of measured properties, is given in Table I. Note that the dry porosity $\phi_{0}$ could be inferred from the mass and volume of a sample determined using TAPPI standard methodologies. The characteristic value $k_{0}=k\left(\phi_{r}\right)$ was estimated from direct measurements of permeability for these pulp samples [26].

\section{B. Imbibition tests}

A typical imbibition experiment was conducted as follows. A reservoir was partially filled with water and placed on a movable stage. A paper sample $\left(10 \mathrm{~cm}\right.$ long, $H_{0}$ wide; see Table I) was secured at its upper edge by a clamp and then positioned above the reservoir. The stage was then raised slowly until the water touched the bottom of the paper and imbibition began. The duration of each experiment was approximately $1000 \mathrm{~s}$. Experiments were conducted at room temperature $\left(T=21 \pm 1^{\circ} \mathrm{C}\right)$ and at a relative humidity of $\mathcal{H}_{\text {rel }}=25 \pm 3 \%$. The progress of the infiltration front was captured using a digital camera (BlackFly 1.3 MP), recording at 14 frames/s (fps) with a spatial resolution of $0.1 \mathrm{~mm} /$ pixel. The images were binarized and the interface position determined through an edge detection algorithm. The wicking height $\ell(t)$ was estimated as the mean height over a horizontal width of $5 \mathrm{~cm}$ and then ensemble averaged over a number of replicate tests.

Our experimental results are summarized in Fig. 5(a), which shows the rise height $\ell(t)$ for each experimental series. The error bars represent the standard deviation over 13 replicates and is representative of all of the data collected, while the shaded area on the left indicates where the data are less certain due to the initial disturbance as the paper is immersed in the reservoir. For comparison, Fig. 5(b) shows a summary of corresponding data taken from the literature.

In order to examine the sensitivity of our results to the ever-present and distracting effects of gravity and evaporation, we carried out some additional experimental tests. To gauge the importance of gravity, we repeated the wicking experiments, but rather than hanging the paper vertically down into the bath, we gently bent the sheet over the side of the tank and let it hang vertically downward. The wicking front $\ell(t)$ from this experiment [magenta line in Fig. 5(a)] is almost indistinguishable from the original experiment, except at early times where the side of the tank affects the spreading. Dimensional analysis suggests that buoyancy effects become important when $\rho g \ell / P_{c}$ becomes order one. For $P_{c}=O\left(10^{7}\right) \mathrm{Pa}$, this requires heights of order $1 \mathrm{~m}$, far greater than the size of our experiment. To assess the impact of evaporation, we determined experimentally a drying rate of $J \approx 10^{-3} \mathrm{~g} / \mathrm{m}^{2}$ s for the paper used. We expect evaporation to be negligible provided that the ratio of evaporation rate per unit width $(2 \ell J / \rho)$ to the volumetric flow rate per unit width created by the advancing front $\left(\phi_{0} H_{0} \dot{\ell}\right)$ is small. With our data, we find that this ratio remains below 5\% 


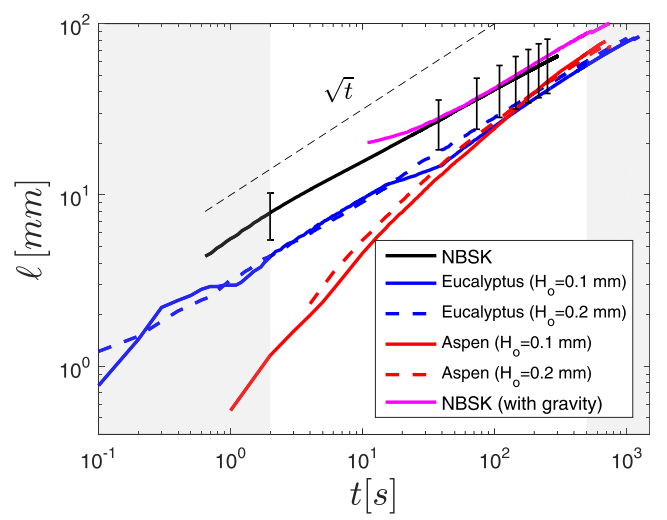

(a)

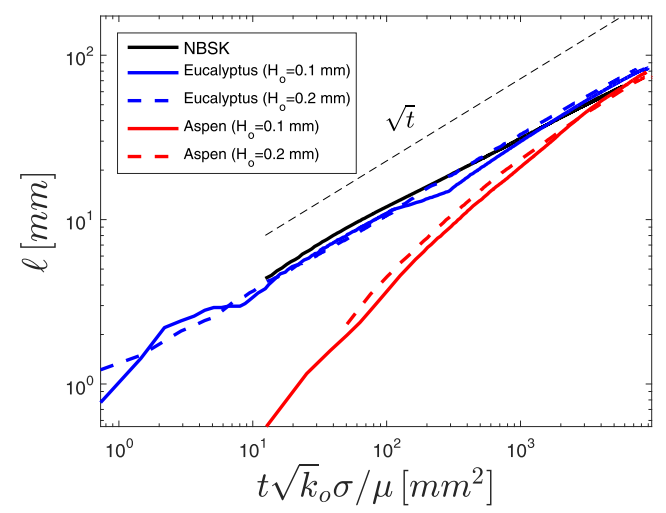

(c)

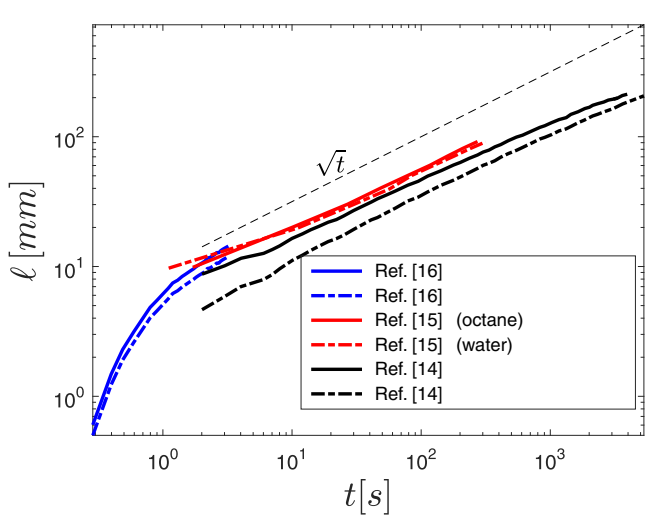

(b)

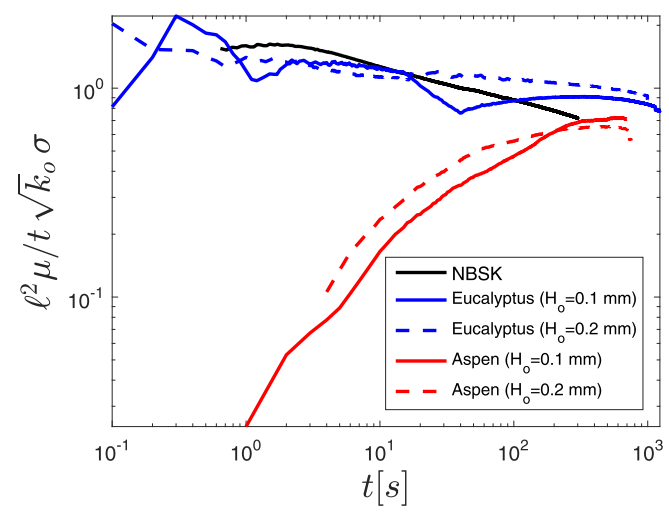

(d)

FIG. 5. Summary of the measurements of the imbibition height $\ell(t)$ : (a) results for the conditions given in Table I and (b) literature results. (c) Our experimental data scaled using the dimensionless time scale in (12) (note that the dimensionless height scale $L_{0}$ was arbitrary in the model). (d) Experimental data replotted to provide an estimate of the applicability of the BCWL (1). The thin dashed lines in (a)-(c) show a scaling of $\sqrt{t}$ for reference.

for $t \lesssim 500 \mathrm{~s}$. At larger times, there is some uncertainty in interpreting the measurements due to evaporation; we have shaded this region in Fig. 5(a).

In an attempt to collapse the data, Fig. 5(c) shows the penetration length against the scaled dimensionless time $t k_{0} P_{c} / \mu$, with $P_{c} \sim \gamma k_{0}^{-1 / 2}$ [cf. (12)]. The NBSK and eucalyptus data collapse onto each other with this scaling and display a scaling very close to, but slightly weaker than, $t^{1 / 2}$. The rise height for aspen, in contrast, does not display a clear power-law behavior: It lies below the other data but increases to approach the same collapsed curve and scaling as the other materials over time. According to the theoretical model, this different behavior can be explained by the relative size of the bulk compressibility of each material, as characterized by the size of the dimensionless parameter $\epsilon$ (see Fig. 3). The rise height for NBSK and eucalyptus appear to evolve with a simple power-law scaling from the earliest times we are able to measure, suggesting a very small value of $\epsilon$, whereas aspen shows a much slower evolution towards a power-law scaling, consistent with a much larger value of $\epsilon$ (i.e., larger bulk viscosity). A similar approach towards the similarity scaling from below can be seen in some of the earlier experimental data presented in Fig. 5(b).

An alternative and more direct comparison of the data with the $t^{1 / 2}$ scaling predicted by the BCLW law (1) is given in Fig. 5(d), which shows a compensated plot of $\ell^{2} \mu / t \sigma \sqrt{k_{0}}$ against time. This 


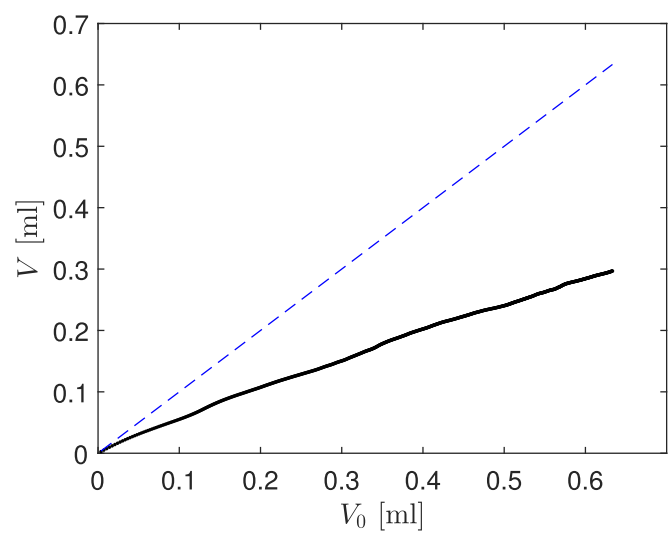

(a)

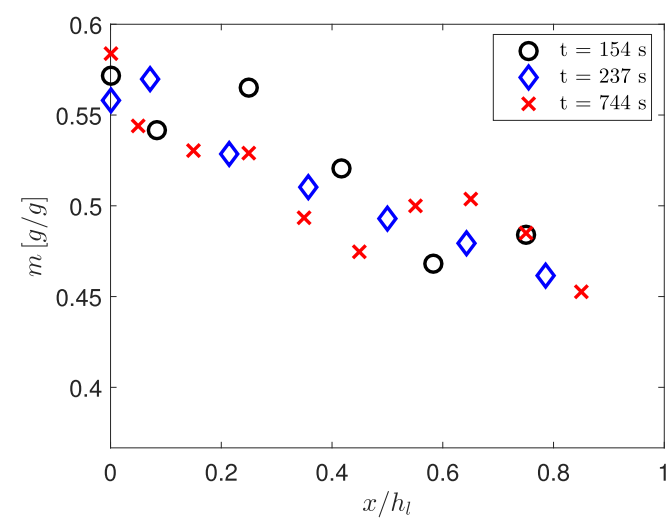

(b)

FIG. 6. Quantification of out-of-plane swelling of paper during imbibition (all result using NBSK paper). (a) Comparison of the reduction in the volume of water in the reservoir $V_{0}$ with the volume $V$ of a column of water of height $\ell$ in paper at its initial porosity $\phi_{0}$ to demonstrate significant swelling during imbibition. (b) Water content profile $m$ determined gravimetrically at three different times.

quantity represents the the coefficient $\alpha$ in the similarity solution in Sec. III A. This plot confirms that all the data for the different materials appear to be approaching a similar value over time. It also suggests a weak subdiffusive behavior in the NBSK and eucalyptus data, which echoes some of the measurements in Fig. 5(b) and is not captured by the theoretical model.

Note that for two of the materials shown in Fig. 5, we conducted experiments with two different initial thicknesses of paper (but the same initial porosity). The data in this figure confirm that the initial thickness has no systematic effect on the rise height, in agreement with the model predictions. ${ }^{1}$

Finally, we conducted two additional tests to gain insight into the imbibition process. The first test was a basic comparison of volumes during imbibition: We compared the total volume of water that had entered the sheet $V_{0}$, determined by the decrease in the volume of the reservoir, with the hypothetical volume of water in the sheet $V=\phi_{0} \ell(t)$ if there were no porosity change. Figure 6(a) shows a significant discrepancy between these quantities, which indicates a change in the average porosity.

The second test provided a destructive approach to estimate the relative change in water content along the swollen sheet. Infiltration was allowed to occur over a set period of time, after which the sample was withdrawn from the water and cut into horizontal strips (i.e., at different $x$ locations). Each wet strip was immediately weighed and the mass of water that had been absorbed was determined. When normalized by the mass of fibers in the strip, this measurement serves as a surrogate for the average porosity. Figure 6(b) shows results for three different infiltration times. Although this was a relatively crude measurement and there is reasonable scatter in the data, there is no systematic difference between the experiments at different times when plotted against $y=x / \ell$, which provides direct evidence that the flow profiles are self-similar over the bulk of the rise.

\footnotetext{
${ }^{1}$ We also conducted some tests in which the paper sheets were first pressed to imprint surface patterns in the manner of an embossing process. This pressing resulted in a spatially varying $\phi_{0}$ and $H_{0}$ but retained a constant initial areal density. Imbibition tests with these sheets gave rise to rise heights $\ell(t)$ that were insignificantly different from those of the uniform sheets, indicating that embossing had no observable effect on imbibition, as expected from the theoretical model, where $\phi_{0}$ and $H_{0}$ appear only in the combination $H_{0}\left(1-\phi_{0}\right)$ [the dimensional equivalent of Eq. (24)].
} 


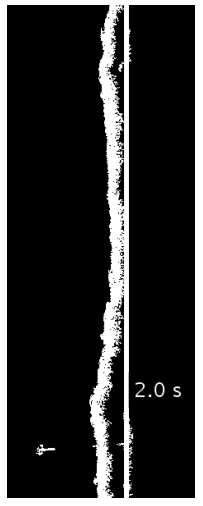

(a)

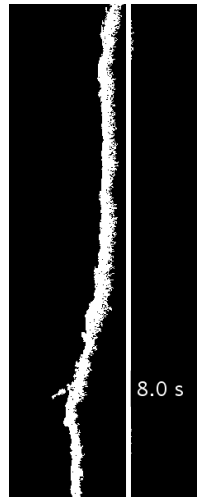

(b)

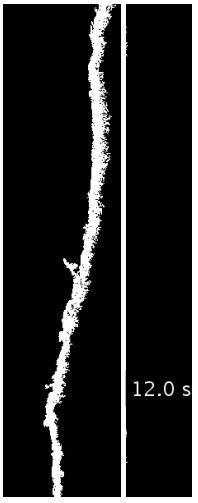

(c)

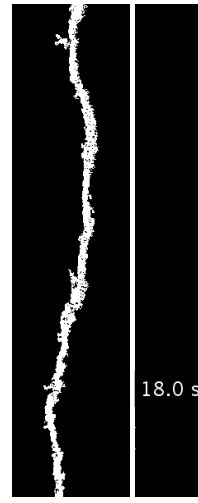

(d)

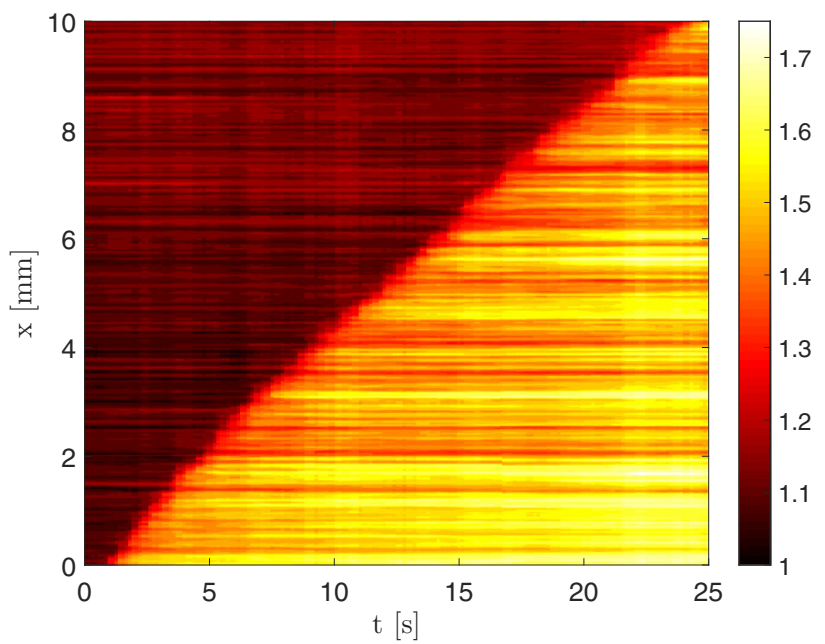

(e)

FIG. 7. Paper thickness during imbibition, as measured by the OCT for (a)-(d) a series of images of the surface of a commercial kitchen towel. The field of view is approximately $1 \mathrm{~mm}$ along the length of the paper. At each time, the position of the binarized upper surface of the paper was acquired through OCT. An additional white line has been drawn in the binarized image to represent the datum. The initial waviness of the surface of the sheet is a crepe, commercially included to effect the mechanical and tactile properties of the paper. (e) Corresponding spatiotemporal plots over the measurement area $[x, t]=[0,10 \mathrm{~mm}] \times[0,25 \mathrm{~s}](\mathrm{NBSK}$, series 1 in Table I).

\section{Out-of-plane deformation}

To measure the swelling of the paper more directly, we used optical coherence tomography (OCT), which provides a measurement of the position of the paper surface, at a high spatial $(10 \mu \mathrm{m} / \mathrm{pixel})$ and temporal (500-fps) resolution [32]. In order to use this technique we required one side of the paper to lie at a fixed position and so we adapted the experimental setup by securing one side of the suspended paper against a plate. Figure 7 shows OCT data of the surface of the sheet during imbibition. The field of view of the tomograph is restricted, with its spatial scan spanning only about $1 \mathrm{~cm}$ of the length of the paper. Individual images taken during each scan and covering a length of about $1 \mathrm{~mm}$ are also shown. Evidently the wetting front, which we see sweeping up the paper surface in the series of images [Figs. 7(a)-7(d)], is comparable to the length of the image $(\sim 1 \mathrm{~mm})$ 

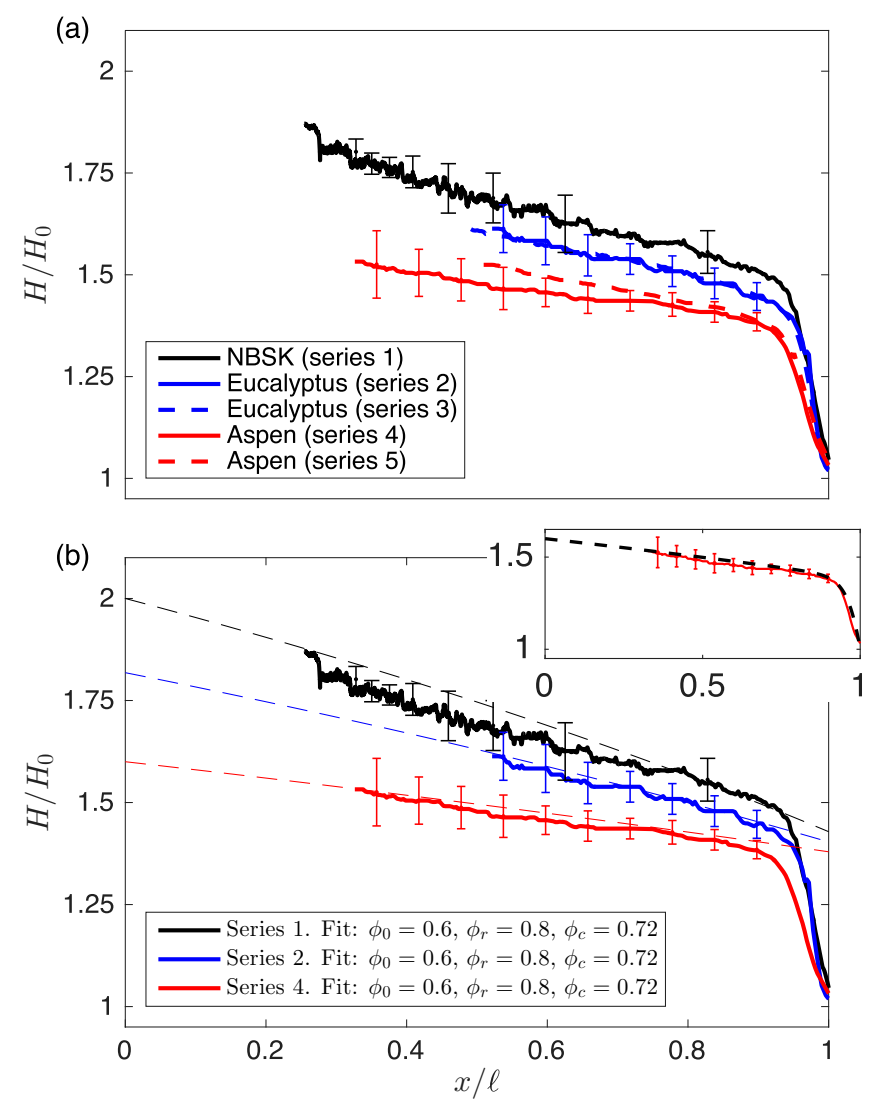

FIG. 8. (a) Spot measurements from the OCT over time at a point $x=10 \mathrm{~mm}$ above the fluid reservoir, as a function of $x / \ell(t)$, for each of the different series in Table I. (b) Some of the same data overlaid by corresponding predictions of the similarity solution (thin dashed line), with parameter choices as marked. The inset shows the data for aspen together with a snapshot from the model with $\epsilon>0$, showing how the boundary layer at the nose can also be captured (specifically, $\epsilon=500$ and dimensionless $t=80$ here).

and thus distinctly longer than the paper thickness $\left(2 H_{0} \approx 0.1 \mathrm{~mm}\right)$. Note also the appearance of fibers rising out of the plane of the surface of the paper during imbibition.

The full scan provides space-time plots of sheet thickness [see the example in Fig. 7(e)], which clearly show the front moving up the paper, as well as highlighting other spatial imperfections that are preserved during the swelling process [horizontal stripes in Fig. 7(e)]. From these data we can extract the thickness $H(t)$ of the paper at any height. By plotting these data as a function of the self-similar coordinate $x / \ell(t)$, we derive a measure of the thickness profiles up the sheet, as shown in Fig. 8(a). This figure shows a sharp gradient in $H$ near the wetting front $(x / \ell \rightarrow 1)$, with a milder swelling of the paper further behind. As with the measurements of rise height $\ell(t)$, the scaled data collapse for paper with different initial thicknesses, in agreement with the predictions of the model.

Indeed, given the estimated values of $\phi_{0}$ and $\phi_{r}$, the latter of which can be estimated from measurements of $m_{r}$ (see Table I) and the density of the fiber, it is possible to draw a more direct comparison with the theoretical model. Figure $8(\mathrm{~b})$ shows fits of the similarity solution $(\epsilon=0)$, which produce broadly the same behavior observed in the OCT measurements. The similarity solution breaks down near the nose, where the gradient in $H$ of the experimental profiles steepens but remains continuous. As noted earlier, the wetting front is at least an order of magnitude wider than the width of the paper sheet and thus the breakdown of the similarity solution there cannot 
simply be associated with the limitations of the shallow-layer framework in the model formulation. Instead, the model suggests that this sort of boundary-layer structure can result from a rate-dependent viscous contribution to the network stress and the inset to Fig. 8(b) verifies that the profile near the nose can be captured by the model when $\epsilon>0$. Indeed, the slope of the profile in the nose region is notably less steep for aspen than for the other materials, which is consistent with the previous observation of the advance of the wicking height that the bulk viscosity for aspen may be higher (such that $\epsilon$ is larger). As a cautionary note, we stress that, since $\phi_{c}$ is a free fitting parameter in these comparisons and since we are just using the simple constitutive functions of Sec. IID, the fits presented here are intended to verify the qualitative predictions of the model rather than to provide a direct quantitative comparison.

\section{CONCLUSION}

In this paper we have provided a combined theoretical and experimental study of imbibition in a thin deformable porous media driven by capillary suction. Our theoretical model explicitly accounts for the out-of-plane swelling of the solid matrix, which, aside from the change in texture due to saturation, is the most visually obvious consequence of imbibition in paper [see Fig. 1(a)]. The model describes two-phase flow in a deformable material in the shallow lubrication limit and incorporates the effect of a strain-rate dependence in the rheological model, which has recently been adopted by Hewitt et al. [26] for the dewatering of pulp suspensions.

In the simplest case with no rate-dependent rheology $(\epsilon=0)$, the model predicts self-similar wetting dynamics, in which the advance of the penetration distance is proportional to $t^{1 / 2}$, as in the classical so-called Washburn (BCLW) law. Interestingly, we found that strain-rate-dependent rheology leads to an initial transient behavior that is not self-similar, but that ultimately the imbibition approaches a self-similar form outside a thin boundary layer at the nose. The rise height initially lags below the self-similar solution $\ell \sim t^{1 / 2}$, but approaches it from below. In practice, the transient approach to self-similar form may last for the duration of an experiment, as we observed in some of our experimental results, which cautions against fitting the Washburn law to observational data at early times.

Overall, our experiments, which used three different cellulose-based sheets, show a number of features in common with model predictions. Perhaps the most significant discrepancy between theory and experiment is the presence of a subdiffusive advance of the wetting front, which has been observed in previous measurements and was very weakly evident in some of our experimental data. Previous research groups have attributed this phenomenon to swelling only. Our work indicates that neither out-of-plane deformation of the medium nor rate-dependent effects in the bulk rheology of the material can give rise to a subdiffusive advance (cf. other nonlinear diffusive problems for which the addition of extra physical ingredients does not prevent the emergence of self-similar behavior, e.g., [33]). As a result, the origin of the subdiffusive behavior still remains an open question.

\section{ACKNOWLEDGMENTS}

Financial support from Kruger Products and the Natural Sciences and Engineering research Council of Canada is gratefully acknowledged. D.M.M. and M.K. would like to thank Vincent Landry, Anna-Karin Ahlman, Marc Fitzgerald, and M. T. Martinez for fruitful and enlightening discussions of absorption in cellulose products and Dr. Andre Phillion for collecting the x-ray microtomographic data.

[1] J. M. Bell and F. K. Cameron, The flow of liquids through capillary spaces, J. Phys. Chem. 10, 658 (1906).

[2] V. R. Lucas, Ueber das zeitgesetz des kapillaren aufstiegs von flüssigkeiten, Kolloid Z. 23, 15 (1918). 
[3] E. W. Washburn, The dynamics of capillary flow, Phys. Rev. 17, 273 (1921).

[4] A. Marmur, Kinetics of penetration into uniform porous media: Testing the equivalent-capillary concept, Langmuir 19, 5956 (2003).

[5] D. M. Cate, J. A. Adkins, J. Mettakoonpitak, and C. S. Henry, Recent developments in paper-based microfluidic devices, Anal. Chem. 87, 19 (2015).

[6] M. A. Hubbe, A. Ayoub, J. S. Daystar, R. A. Venditti, and J. J. Pawlak, Review of cellulosic absorbents, Bioresources 8, 6556 (2013).

[7] M. Landeryou, I. Eames, and A. Cottenden, Infiltration into inclined fibrous sheets, J. Fluid Mech. 529, 173 (2005).

[8] Z. Liu, J. Hu, Y. Zhao, Z. Qu, and F. Xu, Experimental and numerical studies on liquid wicking into filter papers for paper-based diagnostics, Appl. Thermal Eng. 88, 280 (2015).

[9] A. W. Martinez, S. T. Phillips, and G. M. Whitesides, Three-dimensional microfluidic devices fabricated in layered paper and tape, Proc. Natl. Acad. Sci. USA 150, 19606 (2008).

[10] A. W. Martinez, S. T. Phillips, and G. M. Whitesides, Diagnostics for the developing world: Microfluidic paper-based analytical devices, Anal. Chem. 82, 3 (2010).

[11] M. B. Lyne, in Handbook of Physical and Mechanical Testing of Paper and Paperboard, edited by R. E. Mark (Dekker, New York, 1984).

[12] J. A. Bristow, The swelling of paper during the sorption of aqueous liquids, Sven. Papperstidn. 74, 645 (1971).

[13] N. Fries and M. Dreyer, An analytic solution of capillary rise restrained by gravity, J. Colloid Interface Sci. 320, 259 (2008).

[14] S. B. Lindström, N. T. Cervin, and L. Wågberg, in Proceedings of the Fundamental and Applied Pulp and Paper Modeling Symposium (Concordia University, Montreal, 2011).

[15] S. Wiryana and J. C. Berg, The transport of water in wet-formed networks of cellulose fibers and powdered superabsorbent, Wood Fiber Sci. 23, 457 (1991).

[16] D. R. Schuchardt and J. C. Berg, Liquid transport in composite cellulose-superabsorbent fiber networks, Wood Fiber Sci. 23, 342 (1991).

[17] H. Tufvesson, The mechanisms of edge wicking, Ph.D. thesis, Royal Institute of Technology, Sweden, 2006.

[18] P. K. Chatterjee, in Textile Science and Technology, Vol. 7: Absorbency, edited by P. K. Chatterjee (Elsevier, New York, 1985), p. 29.

[19] P. LePoutre, Liquid absorption and coating porosity, Paper Technol. 19, 298 (1978).

[20] R. Masoodi, H. Tan, and K. M. Pillai, Numerical simulation of liquid absorption in paper-like swelling porous media, AIChE J. 58, 2536 (2012).

[21] P. Salminen, Studies of water transport in paper during short contact times, Ph.D. thesis, Åbo Academy, 1988.

[22] J. I. Siddique, D. M. Anderson, and A. Bondarev, Capillary rise of a liquid into a deformable porous material, Phys. Fluids 21, 013106 (2009).

[23] D. M. Anderson, Imbibition of a liquid droplet on a deformable porous substrate, Phys. Fluids 17, 087104 (2005).

[24] J. L. Sommer and A. Mortensen, Forced unidirectional infiltration of deformable porous media, J. Fluid Mech. 311, 193 (1996).

[25] L. Preziosi, D. D. Joseph, and G. S. Beavers, Infiltration of initially dry, deformable porous media, Int. J. Multiphase Flow 22, 1205 (1996).

[26] D. R. Hewitt, D. T. Paterson, N. J. Balmforth, and D. M. Martinez, Dewatering of fibre suspensions by pressure filtration, Phys. Fluids 28, 063304 (2016).

[27] D. R. Hewitt, J. A. Neufeld, and N. J. Balmforth, Shallow, gravity-driven flow in a poro-elastic layer, J. Fluid Mech. 778, 335 (2015).

[28] R. Buscall and L. R. White, The consolidation of concentrated suspensions. I. The theory of sedimentation, J. Chem. Soc. Faraday Trans. 1, 873 (1987).

[29] A. Kulachenko, in Mechanics of Paper Products, edited by K. Niskanen (De Gruyter, Berlin, 2012). 
[30] G. W. Jackson and D. F. James, The permeability of fibrous porous media, Can. J. Chem. Eng. 64, 364374 (1986).

[31] Y. Sharma, A. B. Phillion, and D. M. Martinez, Automated segmentation of wood fibres in micro-CT images of paper, J. Microsc. 260, 400 (2015).

[32] T. Fabritius, R. Myllylä, S. Makita, and Y. Yasuno, Wettability characterization method based on optical coherence tomography imaging, Opt. Express 18, 22859 (2010).

[33] R. A. Wooding, Convection in a saturated porous medium at large Rayleigh number or Peclet number, J. Fluid Mech. 15, 527 (1963). 